\title{
Lumen
}

Selected Proceedings from the Canadian Society for Eighteenth-Century Studies

\section{Les tableaux dans le Manuscrit trouvé à Saragosse et dans Pauliska, ou La perversité moderne}

\section{Natalie LaFleur}

Volume 33, 2014

URI : https://id.erudit.org/iderudit/1026564ar

DOI : https://doi.org/10.7202/1026564ar

Aller au sommaire du numéro

Éditeur(s)

Canadian Society for Eighteenth-Century Studies / Société canadienne d'étude du dix-huitième siècle

ISSN

1209-3696 (imprimé)

1927-8284 (numérique)

Découvrir la revue

Citer cet article

LaFleur, N. (2014). Les tableaux dans le Manuscrit trouvé à Saragosse et dans Pauliska, ou La perversité moderne. Lumen, 33, 41-53.

https://doi.org/10.7202/1026564ar d'utilisation que vous pouvez consulter en ligne. 


\title{
Les tableaux dans le Manuscrit trouvé à Saragosse et dans Pauliska, ou La perversité moderne
}

\author{
Natalie LAFLeur \\ Université de Montréal / Université Paris-Sorbonne
}

Durant la seconde moitié du XVIII ${ }^{\mathrm{e}}$ siècle, les échanges entre le théâtre et le roman se multiplient. Le théâtre influence le roman par le biais de ses sujets, de ses personnages et de ses intrigues. La fréquentation mondaine du théâtre est mentionnée dans plusieurs romans tels que Les liaisons dangereuses et Le neveu de Rameau. Les romanciers s'amusent à faire jouer à leurs personnages des scènes de comédies. Le théâtre occupe progressivement une place encore plus importante dans le roman du Tournant des Lumières, où il ne se contente plus de mimer des personnages stéréotypés ou des scènes de pièces de théâtres populaires. Les auteurs adoptent désormais un cadre scénique vivant et sensible du théâtre: le tableau.

Le concept de tableau a tout d'abord été conçu par Denis Diderot pour ses drames. Bien qu'il n'ait pas inventé le mot, c'est lui qui l’a théorisé le mieux dans ses «Entretiens sur Le Fils Naturel ». Selon lui, un tableau est une "disposition de [...] personnages sur la scène, si naturelle et si vraie, que, rendue fidèlement par un peintre, elle me plairait sur la toile ${ }^{1}$. Non seulement Diderot aime ce type de scène pittoresque, mais il l'envisage comme un critère essentiel pour la réussite d'une pièce. D'autres auteurs du XVIII ${ }^{\mathrm{e}}$ siècle tels que Richardson et Sade sont aussi loués pour leur art de composer des tableaux. Comme

1. Denis Diderot, «Entretiens sur Le fils naturel», 1757, dans (Euvres complètes. Le drame bourgeois, éd. Jacques Chouillet et Anne-Marie Chouillet, Paris, Hermann, t. 10,1980 , p. 80. 
l'a déjà souligné Catherine Ramond, les études sur le tableau dans les romans se limitent souvent à un romancier ${ }^{2}$. De plus, les recherches dans ce domaine sont dans la plupart des cas restreintes à un genre de tableau. Deux types de tableaux feront l'objet de la présente analyse et ce, dans deux romans, Pauliska, ou La perversité moderne de JacquesAntoine Révéroni Saint-Cyr et le Manuscrit trouvé à Saragosse de Jean Potocki. Dans ces deux textes, les évènements sont racontés par plus d'un personnage; la voix narrative de Pauliska est divisée entre deux héros, alors que le Manuscrit trouvé à Saragosse est un récit polyphonique. Ils se rejoignent par leur maniement des tableaux, notamment dans les scènes de débauche et de merveilleux.

Les publications des romans de Révéroni Saint-Cyr et Potocki ainsi que la théorie des tableaux de Diderot seront d'abord rappelées. Il s'agira de voir comment la mise en relief des tableaux libertins est appuyée d'une part par les pantomimes et, d'autre part, par la sonorité, afin de rendre la théâtralité du tableau. Les tableaux fantastiques tout comme les tableaux libertins comptent sur la dimension sonore pour créer l'ambiance. Par contre, dans les tableaux fantastiques, les effets fantasmagoriques sont définis par un jeu de lumière particulier.

\section{La publication des romans et une brève explication du tableau}

Avant d'aborder l'analyse des tableaux, il est important de rappeler le sujet et la genèse des romans. Pauliska, ou La perversité moderne, publiée en 1796, se déroule entre 1793 et 1795, au moment de l'envahissement de la Pologne par Catherine II. L'héroïne se réfugie en Hongrie, côtoyant les pires violences et des lieux obscurs tels que des montagnes, des grottes et des souterrains. Le récit est marqué par l'imaginaire, des illusions de spectre et des outils de séduction. Révéroni Saint-Cyr emploie le mot «moderne» dans son titre puisque Pauliska et son amant Édouard abordent des inventions modernes comme le magnétisme et l'inoculation, dont se servent des êtres méchants pour leur propre plaisir sexuel. En 1798, le rédacteur des Veillées de Muses écrit dans le compte rendu qu'il consacre à Pauliska : «Ses tableaux sont bien affligeants pour l'espèce humaine, s'il est vrai qu'elle puisse tom-

2. Catherine Ramond, Roman et théâtre au XVIII siècle: le dialogue des genres, SVEC, 4, 2012, p. 2-3. 
ber dans cet état de corruption qui ne lui laisse plus d'objets de choix qu'entre le bizarre et le monstrueux ${ }^{3}$ ». De plus, «[1]'auteur ne connaît l'art des gradations que dans ses tableaux ${ }^{4} »$.

L'historique de la publication du Manuscrit trouvé à Saragosse est plus complexe. Jean Potocki a beaucoup remanié son roman, rédigeant une partie de son œuvre de 1793 à 1803 et continuant de l'augmenter peu à peu jusqu'en 1812. L'auteur a publié deux contes tirés du récit à Paris en 1813, Avadoro, histoire espagnole et Dix Journées de la Vie d'Alphonse Van Worden. Le Manuscrit trouvé à Saragosse a aussi été traduit en allemand en 1809 et en polonais en 1847 . Après qu'Edmond Chojecki a publié la traduction polonaise, faite d'après un manuscrit, il l'a détruit. Par la suite, le roman est tombé dans l'oubli en France. Le public français ne découvre l'auteur qu'en 1958, grâce à Roger Caillois, qui publie un quart du roman. L'édition de René Radrizzani publiée en 1989 tente de restituer l'œuvre, en s'appuyant sur des imprimés, des autographes et des copies manuscrites du roman, ainsi que sur la traduction de Chojecki ${ }^{5}$. Le personnage d'Alphonse, entraîné dans une étrange aventure, doit subir des épreuves avant de se voir confier un grand secret. L'auteur mélange plusieurs genres de contes: picaresques, philosophiques, libertins et fantastiques; tout comme Révéroni Saint-Cyr, il peint des tableaux.

Il est essentiel de rappeler la théorie du tableau conçue par Diderot et étudiée par Pierre Frantz afin de comprendre l'esthétique qui lie les différents types de tableaux libertins et fantastiques. Pour Diderot, un tableau doit mettre en relief tous les éléments scéniques, incluant les décors, les objets, les indications de jeux (les gestes et les attitudes). Diderot, au théâtre comme dans la peinture, cherche à être ému. Dans Lesthétique du tableau dans le théâtre du XVIII siècle, Pierre Frantz précise aussi que le tableau peut faire voir une émotion forte qui s'intensifie. Il nomme cette gradation d'un flux d'énergie le tableau-comble.

Un tableau-comble est né au croisement de l'énergétique sensualiste de Diderot et de l'esthétique du sublime. Il organise la plupart du

3. Compte rendu dans Les veillées des muses, 10, 1798, reproduit dans Pauliska, ou La perversité moderne: Mémoires récents d'une Polonaise, de Jacques-Antoine Révéroni Saint-Cyr, 1796, Paris, Desjonquères, coll. «XVIII siècle», 1991, p. 214.

4. Ibid., p. 219.

5. Jean Potocki, Manuscrit trouvé à Saragosse, 1813, édition de René Radrizzani, Paris, Librairie générale française, coll. «Le livre de poche», 2007. 
temps l'ensemble des personnages autour d'une pantomime centrale, souvent silencieuse, qui manifeste un comble du pathétique ou un comble du sublime ${ }^{6}$.

Cette composition picturale et sensorielle est présente dans les livres de Diderot La religieuse et Le neveu de Rameau grâce aux pantomimes. Diderot décrit minutieusement les gestes dans les tableaux libertins de manière que la scène s'intensifie de plus en plus.

\section{Les tableaux libertins}

Dans les tableaux libertins, pour rendre cet accroissement d'énergie, Jean Potocki et Révéroni Saint-Cyr utilisent l'expressivité du corps. Vers la fin du Manuscrit trouvé à Saragosse, Potocki livre un de ses plus longs et plus dramatiques tableaux ${ }^{7}$. Il s'agit de l'épisode où Torres rend visite à madame Paduli, qui s'amuse en prétendant être souffrante afin de le séduire, non parce qu'elle l'aime, mais afin d'imiter les actrices de Londres qu'elle admire ${ }^{8}$. Potocki met l'accent sur les mains, sur les regards ainsi que sur les larmes de la comtesse:

[Madame Paduli] me tendit sa main blanche comme neige et me transperça le regard, les yeux pleins de larmes et, d'une voix tremblante, me fit quelques mots dont j'eus de la peine à saisir le sens. Je levai les yeux sur la malade. Comme elle était belle avec cette pâleur! Une douleur intérieure convulsait ses traits, mais un sourire d'ange se dessinait sur ses lèvres. La même femme, si saine et si gaie il y a quelques jours, s'approchait aujourd'hui du tombeau. C'était donc moi, l'indigne qui avait coupé cette fleur dans son épanouissement, c'était donc moi qui avais jeté tant de charmes dans l'abîme! Cette idée me glaça le cœur et me remplit de remords inexprimables. Je pensai donc pouvoir sauver sa vie par quelques paroles; je me jetai à ses genoux devant elle et portai sa main à ma bouche. La marquise pencha sa tête sur mon cou, ses boucles se répandirent sur mon visage et, poussé par un sentiment

6. Pierre Frantz, L'esthétique du tableau dans le théâtre du XVIII siècle, Paris, Presses universitaires de France, coll. «Perspectives littéraires», 1998, p. 168.

7. Il existe aussi quelques petits tableaux libertins mettant en scène Alphonse, Elmina et Zibeddé. Par exemple : «Zibeddé se rappela le rôle d'amante qu'elle avait autrefois étudié avec sa sœur. Celle-ci voyait dans mes bras l'objet de ses feintes d'amours et livrait ses sens à cette douce contemplation. La cadette, souple, vive, brûlante, dévorait par le tact et pénétrait par ses caresses.»(Jean Potocki, op. cit., p. 79).

8. Ibid., p. 441. 
incompréhensible, je lui avouai que je l'aimais. Je ne sais comment j’oubliai Elvire, bien que sa pensée revînt toujours dans mon cœur; d'autre part, je ne pouvais permettre que la marquise mourût. En effet, je n'avais de ma vie vu de femme aussi charmante.

«Dieu d'amour! s'écria l'Italienne. Celui que j’aimais m’a rendue à la vie $e^{9}$ »

Il s'agit d'un tableau-comble, puisque l'intérêt de la scène est centré sur madame Paduli et l'émotion du personnage parvient à s'élever à son comble. L'élément le plus important de ce tableau libertin est son évolution émotive. Potocki décrit la faiblesse de madame Paduli et ensuite s'applique à décrire la manière dont elle se réanime. Lorsqu’elle manque d'énergie, elle ne fait que tendre sa main à Torres. Plus loin, elle gagne de la vigueur et porte elle-même la main à la bouche de Torres. La plus grande partie de la scène se passe en silence, à part des interruptions causées par la voix de madame Paduli. Selon Pierre Frantz, cette impossibilité de parler est typique des tableaux-combles des pièces de théâtre. Dans certains romans comme La religieuse, Diderot peint beaucoup de tableaux énergiques muets. Cependant, le tableau de Potocki est différent parce que le personnage central, madame Paduli, feint sa maladie, alors que, chez Diderot, il n'y a pas cette dimension du mensonge. Diderot décrit un tableau-comble muet quelques moments avant la mort de madame de Moni. La supérieure est entourée de religieuses et semble n'avoir plus de forces quand, tout à coup, elle se réanime, prononce quelques paroles comme si elle était en santé et puis meurt ${ }^{10}$. Bien que l'épisode puisse paraître un peu affecté, le soupçon disparait lorsque la supérieure expire immédiatement après son discours. L'agonie de Madame de Moni n'était pas simulée.

Suzanne décrit un deuxième tableau centré sur un personnage expirant. L'épisode donne à voir les derniers moments de son amie sœur Ursule:

Ensuite, pressentant que j’allais la perdre, et persuadée que sa maladie était une suite ou de la mienne, ou de la peine qu'elle avait prise, ou des soins qu'elle m’avait donnés, je me mis à pleurer et à me désoler de toute

9. Ibid., p. 433.

10. Voir Denis Diderot, La religieuse, 1780, édition de Robert Mauzi, Paris, Gallimard, coll. «Folio classique», 2004, p. 86-87. 
ma force. Je lui baisai le front, les yeux, le visage, les mains [...] Cependant, elle était comme distraite, elle ne m'entendais pas; une de ses mains se reposait sur mon visage et me caressait; je crois qu'elle ne me voyait plus [...] Elle fit un effort pour me présenter les joues; je les baisai, elle me prit la main, [...] il semblait qu'elle ne voulait pas, qu'elle ne pouvait me quitter [...] la pauvre sœur n'était plus ${ }^{11}$.

D’une part, l'épisode se rapproche du tableau de madame Paduli du Manuscrit trouvé à Saragosse, grâce au mélange de léthargie et d'affection; Diderot et Potocki combinent tous deux les signes de sensibilité, les larmes et les baisers. D'autre part, le tableau de sœur Ursule est différent de Potocki, car l'agonie du personnage n'est pas feinte.

Dans le tableau-comble du Manuscrit trouvé à Saragosse, on voit la progression d'énergie non seulement dans les actions de madame Paduli, mais aussi dans ses paroles. Au début du tableau, la marquise ne dit rien, mais une fois que son émotion parvient à son comble, l'enthousiasme lui arrache une exclamation.

L'emplacement de ce tableau dans l'œuvre mérite aussi d'être examiné. L'éditeur, René Radrizzani, a choisi d'inclure ce long tableau dans une note de bas de page. Le tableau décrit dans cette version du texte fait seulement six lignes alors que la variante, notée en bas de page, occupe un paragraphe. La version plus courte du tableau peignant madame Paduli est la suivante:

Elle était dans son lit et me tendit la main; elle était brûlante, ce que je crus être un effet de la fièvre. Je levai les yeux sur la malade et je la vis plus qu'à demi nue. Jusqu'alors, je n'avais connu des femmes que le visage et des mains. Ma vue se troubla, mes genoux faiblirent. J'étais infidèle à Elvire, sans même savoir comment cela m'était arrivé. «Dieu d'amour! s'écria l'Italienne, voilà de tes miracles! Celui que j'aimais m'a rendue à la vie ${ }^{12}$ !»

La version la plus courte laisse croire que madame Paduli a des symptômes de fièvre causée par son désir sexuel, alors que la plus longue version s'attarde davantage sur son état léthargique plutôt que sur ses pantomimes libertines.

Les scènes de débauche dans Pauliska, ou la perversité moderne sont décrites plus minutieusement que chez Potocki. Le tableau-comble le

11. Ibid., p. 169-170.

12. Jean Potocki, op. cit., p. 433. 
plus frappant de Révéroni Saint-Cyr se trouve aussi vers la fin du roman, dans un épisode sadique qui a lieu chez la tante de Julie. Le prêtre veut que les trois femmes (mademoiselle Brunher, Julie et Pauliska) le frappent avec un cilice afin de se faire pardonner ses péchés. La tante de Julie le frappe en premier. Puis, la scène s'intensifie avec les cris de l'abbé quand ses deux autres complices le punissent à son gré.

«Frappez, mes sœurs, frappez un pécheur», s'écria-t-il en redoublant de chaleur et nous donnant à chacune un des fouets mystérieux.

La bonne $\mathrm{M}^{\text {lle }}$ Brunher frappait bénignement et à chaque coup marmottait une oraison. Le père trépignait d'impatience en répétant, à haute voix, les versets du psaume. «Plus fort! plus fort! s'écriait-il avec rage, en s'adressant à nous: frappez, anges d'Héliodore, frappez de verges un impie comme lui. » Son visage, pâle en tout temps, se couvrait alors d'une rougeur brûlante, il se précipitait au-devant de nos coups, ses yeux enivrés semblaient aspirer au ciel. Julie et moi, nous frappions à souhait et nos bras commençaient à se fatiguer, lorsque, par un bienfait de la pénitence sans doute, l'abbé tomba sur le parquet tremblant de tous ses membres, dans un état d'ivresse évident, et s'écriant: «Le ciel me pardonne, mes sœurs, une main de feu m'inscrit au livre de vie. Je ressens l'avant-goût des jouissances célestes ${ }^{13}$ !»

Cette scène de mortification correspond à la définition du tableau de Diderot, évoqué par Dorval dans Le fils naturel, car elle fait une place essentielle au jeu muet, au dispositif pictural et intervient à un moment où l'intensité de l'action et de l'émotion atteint son apogée ${ }^{14}$. Lorsque l'abbé tombe en tremblant de tous ses membres, son ivresse est à son comble. Cette scène correspond bien au tableau, car Révéroni SaintCyr décrit les différentes figures, en respectant toutefois le cadre du décor et l'unité de l'intérêt ${ }^{15}$. L'auteur décrit mademoiselle Brunher frappant le prêtre, alors que Pauliska les observe; le plan-séquence s'attarde ensuite sur le prêtre. L'auteur signale la présence d'une troisième femme, Julie, et la transformation du rôle de Pauliska, qui passe de spectatrice à actrice. Même si l'auteur distingue les actions des

13. Jacques-Antoine Révéroni Saint-Cyr, Pauliska, ou La perversité moderne: Mémoires récents d'une Polonaise, 1796, Paris, Desjonquères, coll. "XVIII siècle», 1991, p. 143.

14. Pierre Frantz, op. cit., p. 155.

15. Ibid., p. 167. 
personnages, il maintient l'unité du tableau. L'épisode se déroule entièrement dans le même décor et est centré autour de la figure du prêtre en proie à la plus grande jouissance.

Le tableau met en évidence des expressions faciales qui, au théâtre, ne seront pas nécessairement perçues par les spectateurs et qui, de plus, peuvent être difficiles à restituer lors d'une représentation - un visage qui rougit, par exemple. Ces descriptions agissent comme les didascalies au théâtre : elles peuvent être repérées par les lecteurs de la version écrite, mais pas nécessairement par les spectateurs. L'épisode rappelle Justine ou Les infortunes de la vertu du marquis de Sade où les mêmes types de personnages - un prêtre libertin et quelques femmes — sont mis en scène.

Potocki et Révéroni Saint-Cyr ne cherchent pas à décrire uniquement des tableaux libertins, mais ils peignent surtout des tableaux fantastiques. Il s'agit de scènes où de «fausses images [sont] produites dans l'intention de tromper le sujet de la perception ${ }^{16} »$. Ces deux auteurs montrent un personnage central dans des épisodes pleins de vivacité.

\section{Les tableaux fantastiques}

Dans Pauliska, ou La perversité moderne, l'héroïne se met à croire en deux occasions qu'elle est entourée de fantômes dans un lieu souterrain. Dans le premier épisode, Pauliska entend des voix mystérieuses. Elle s'imagine qu'il s'agit de fantômes et qu'elle se trouve aux portes de l'enfer:

Je descendis la première marche, une seconde, puis une troisième: prête à mettre le pied sur la quatrième, j’entends au fond de l'abîme des voix; on parlait bas, je ne pouvais rien distinguer; mais je frissonnai et ma main fût prête à lâcher le bloc où je me retenais. J'essayai néanmoins de reprendre courage et de rappeler ma respiration; je crus ne voir qu'un jeu de mon imagination, et je tâchais de me raffermir sur mes pieds chancelants, lorsque tout à coup une grande clarté paraît au loin sous moi, plusieurs fantômes noirs s'écrient: Recevons-la! Je crois voir l'enfer

16. François Rosset et Dominique Triaire, De Varsovie à Saragosse: Jean Potocki et son œeuvre, Louvain, Éditions Peeters, coll. «République des lettres », 2000, p. 263. 
entrouvert; mes genoux faiblissent, mes mains s'ouvrent, lâchent le bloc, et je tombe $[. .$.$] au fond du gouffre... { }^{17}$

D'abord, elle entend les voix au loin, puis les distingue peu à peu et s'évanouit en pensant avoir entendu des «fantômes ». Une fois réveillée, elle constate que la source de la lumière est une lampe qui éclaire le sous-terrain et que les voix sont celles de plusieurs familles de réfugiés $^{18}$. Dans un deuxième épisode, l'absence de présences humaines est ce qui effraie Pauliska ${ }^{19}$.

La lumière particulière qu'on retrouve dans cet épisode évoque la technique picturale du clair-obscur en peinture. Dans l'Encyclopédie ou Dictionnaire raisonné des sciences, des arts et des métiers, Paul Landois écrit dans la première partie de la définition:

En peinture, la connaissance de la lumière, par rapport à la distribution qu'on en doit faire sur les objets, est une des plus importantes parties \& des plus essentielles à cet art. Elle contient deux choses, l'incidence des lumières \& des ombres particulières, \& l'intelligence des lumières en général, que l'on appelle ordinairement le clair obscur ${ }^{20}$.

Dans Pauliska, l'auteur peint l'ombre de son héroïne et un espace souterrain à demi-éclairé. Ces jeux d'ombres et de lumières font souvent partie des tableaux quasi surnaturels d'où l'on voit surgir des fantômes dans le roman de Jean Potocki.

Chez ce dernier, la théâtralité des tableaux fantastiques se voit en plus appuyée par le langage, puisqu'il s'agit de récits emboîtés qui sont transmis à l'oral. Le chef bohémien dans le Manuscrit trouvé à Saragosse décrit un épisode nocturne étrange, où il est enfermé dans une prison; il aperçoit dans le cimetière des hommes et il entend ensuite les gémissements de trois spectres. Ce n'est pas l'unique cas où

17. Jacques-Antoine Révéroni Saint-Cyr, op. cit., p. 70-71.

18. Ibid., p. 74-75.

19. Ce tableau est plus bref que les autres tableaux fantastiques dans Pauliska. Une autre différence tient dans ce qu'on n'y relève aucune présence de fantômes.

Arrivés dans la grotte, quelle est ma surprise! Aucune lumière, plus de voix humaine, nul indice du séjour des colons... «Grand Dieu! ils ont tous péri!» m’écriai-je désespérée, j’avance, je heurte un corps, je vais tomber loin de là sur des vêtements: mon imagination me peint mes compagnons morts, dévorés, anéantis; je ne vois plus que des ossements. [...] je me crois seule au monde [...] (Ibid., p. 78).

20. Encyclopédie ou Dictionnaire raisonné des sciences, des arts et des métiers, édité par Jean Le Rond d'Alembert et Denis Diderot, Paris, 1751-1772, t. 3, p. 499. 
un personnage est confronté à un phénomène qui remet en question sa perception ${ }^{21}$.

Le chef bohémien raconte également l'histoire du chevalier de Tolède qui a lui aussi cru voir un fantôme. Un soir, l'ami du chevalier, Aguilar, lui rend visite afin de l'avertir qu'il a le pressentiment de sa mort imminente. Aguilar promet au chevalier de l'informer de l'existence d'un purgatoire s'il meurt. La scène se passe à minuit. Le décor terrifiant est accentué par un orage. Le chevalier entend quelqu'un frapper au volet de sa maison et il est convaincu que celui qui frappe ne peut être nul autre que son ami Aguilar. Le tableau s'achève avec un gémissement.

Tolède se levait, regardait à sa montre, et puis il retournait à son lit et pleurait. La nuit était sombre, la lueur de quelques éclairs lointains brillait à travers des ais de nos volets. Lorage se rapprocha et ses terreurs ajoutèrent à la tristesse de notre situation. Minuit sonna et nous entendîmes frapper trois coups à notre volet.

Tolède ouvrit le volet et dit: «Es-tu mort?»

«Je suis mort», répondit une voix sépulcrale.

«Y a-t-il un purgatoire?» dit Tolède

«Il y en a un; et j'y suis », répondit la même voix, et puis nous entendîmes comme un gémissement douloureux.

Tolède tomba, le front prosterné dans la poussière ${ }^{22}$.

Ce deuxième tableau fantasmagorique de Potocki repose entièrement sur deux voix. Le chevalier ne voit pas la silhouette du «fantôme». Lauteur explique plus tard que l'homme qui avait ouvert la fenêtre du chevalier s'était trompé de maison. Il avait monté une échelle appuyée contre la maison et il était tombé. Le tableau d'Aguilar est plus comique et repose davantage sur le dialogue que les autres épisodes fantastiques du roman.

Potocki, tout comme Révéroni Saint-Cyr décrivent les illusions de spectres et l'effet qu'ils produisent sur le personnage. Ils éclaircissent plus loin dans leurs récits comment le trompe-l'œil a été créé, en précisant dans chacun des cas qu'ils ne s'agissaient pas réellement de fantômes. Ces jeux d'apparences trompeuses ne sont pas restreints aux tableaux fantasmagoriques. Dans Pauliska, ou La perversité moderne,

21. François Rosset et Dominique Triaire, op. cit., p. 256.

22. Jean Potocki, op. cit., p. 345. 
Révéroni Saint-Cyr utilise cette même technique narrative pour décrire les inventions dans le roman. Les personnages décrivent les effets des inventions dans un cadre fantastique et a posteriori dissipe la tromperie, en expliquant tous ses mécanismes. Pauliska est forcée à imprimer des assignats pour des faux-monnayeurs et doit mouvoir le balancier de la presse. S'appuyant sur la machine, elle entend un gémissement. Les ouvriers lèvent le mécanisme, exposant son collègue Durand écrasé, étranglé par la corde de l'imprimerie et elle croit l'avoir tué23. Plus tard, Pauliska se rend compte que Durand n'est pas mort et Durand lui-même lui explique comment elle a été dupée par l'illusion. Après qu'un homme lui eut lié les mains et les pieds à la presse, un second homme de sa connaissance, faisant preuve d'une certaine sensibilité, était venu pour desserrer les cordes. Sous la pression de la machine, ses yeux s'étaient fermés parce qu'il s'était évanoui. Henri Lafon qualifie cette technique narrative, où on décrit le côté fantastique, l'effet d'émerveillement de la machine parlante et où on diffère l'explication mécanique comme «le double romanesque de l'époque ${ }^{24}$ ». Ce double rôle est également visible dans les inventions et les accessoires des scènes libertines qui ponctuent le roman ${ }^{25}$. Cette confusion entre le vrai et le faux est mise en relief autant chez Révéroni Saint-Cyr que chez Potocki.

D’ailleurs, vers la fin de son roman, Potocki élucide la plupart des tableaux irréels représentés dans le Manuscrit trouvé à Saragosse et il fournit la clé des épisodes les plus mystérieux. Cependant, il introduit aussi le personnage du diable. Celui-ci n'est jamais déchiffré ni expliqué de manière rationnelle et garde jusqu’à la fin ses traits surnaturels.

La nuit agit comme un cadre idéal pour le fantasme et, la plupart du temps, le personnage qui vit une expérience anormale est seul. Nul

23. Jacques-Antoine Révéroni Saint-Cyr, op. cit., p. 107-108.

24. Lafon, Henri, «Machines à plaisir dans le roman français du Xviır siècle ", Revue des sciences humaines, 186-187, 1982, p. 111-120.

25. Par exemple, le fauteuil du baron d'Olnitz a un mécanisme qui permet le libertinage en contraignant la victime : «[le baron d'Olnitz] me repoussa froidement dans un fauteuil dont les bras se croisèrent devant moi par des ressorts et disparut encore plus rapidement qu'à l'ordinaire. » (Jacques-Antoine Révéroni Saint-Cyr, op. cit., p.67) Pauliska décrit l'effet de l'invention. Henri Lafon a déjà mentionné l'idée de vouloir prendre le vrai pour le faux, avec l'objet trompeur du sofa à ressort, mais dans Les Erreurs Instructives ou Mémoires du Comte de **** (1765), de Jonval, dans Henri Lafon, «Machines à plaisir dans le roman français du XVIII siècle », Revue des sciences humaines, 186-187, 1982, p. 113. 
ne peut confirmer ou nier ce qu'il a vu. Potocki s'amuse à décrire des scènes trompeuses afin de démontrer à quel point l'imagination est une faculté puissante. Les épisodes fantastiques dans le Manuscrit trouvé à Saragosse rappellent la définition de l'adjectif «imaginaire » que propose Diderot dans l'Encyclopédie: "Que la chose soit ou ne soit pas comme je l'imagine, je souffre ou je suis heureux; ainsi l'imaginaire peut être dans le motif, dans l'objet; mais la réalité est toujours dans la sensation ${ }^{26}$.» Aucun fantôme n'apparaît dans les tableaux de Potocki et de Révéroni Saint-Cyr; c'est le cadre nocturne et les voix inconnues qui peignent l'idée d'un spectre dans l'esprit des personnages

Dans une scène plus agitée, Potocki présente un deuxième tableau fantastique dans lequel le pèlerin maudit croit entendre la voix sépulcrale de son père:

La nuit vint; le ciel se chargea de nuages; un tourbillon soudain ouvrit ma fenêtre; un éclair bleuâtre sembla parcourir ma chambre et la laissa ensuite plus sombre qu'elle n'était auparavant. Au milieu de cette obscurité, je crus distinguer quelques formes fantastiques, ensuite, il me sembla entendre le corps de mon père pousser un long gémissement, que les échos lointains répétèrent à travers l'espace de la nuit. Je voulus me lever, mais j'étais retenu à ma place, et dans l'impossibilité de faire aucun mouvement. Un froid glacial pénétra mes membres; j’eus le frisson de la fièvre, mes visions devinrent des rêves et le sommeil s'empara de mes sens ${ }^{27}$.

Ce tableau fantastique est un peu différent des autres que l'on trouve chez Potocki, car les phénomènes naturels sont décrits de manière plus détaillée ${ }^{28}$. Néanmoins, cette scène d'apparition, comme les deux précédentes, met en relief le décor de la scène. La voix fantasmagorique est en plus multipliée par ses échos. Cet épisode accentue aussi davantage la sensibilité du personnage que dans le tableau d'Aguilar. Le pèlerin maudit décrit non seulement les propriétés acoustiques,

26. Encyclopédie ou Dictionnaire raisonné des sciences, des arts et des métiers, op. cit., t. 8 , p. 560.

27. Jean Potocki, op. cit., p. $49^{8}$.

28. Dans une variante, l'auteur avait ajouté des météores afin d'intensifier la scène. «La nuit vint; le ciel se chargea de nuages; un tourbillon soudain ouvrit ma fenêtre; un éclair bleuâtre sembla parcourir ma chambre [...]. Au milieu de cette obscurité, je crus distinguer quelques formes fantastiques et l'air me parut rempli de météores singulièrement figurés». (Jean Potocki, op. cit., p.498, n. 18) 
mais aussi les symptômes physiques déclenchés par la voix mystérieuse. Il se rend compte par la suite que cette voix, cette manifestation qu'il croyait être le fantôme de son père, n'était en fait qu'un inconnu qui avait pénétré dans la chambre.

Les tableaux libertins et fantastiques reposent sur des mises en scène différentes dans Pauliska et dans le Manuscrit trouvé à Saragosse. Les deux auteurs s'amusent à peindre des scènes de fiction créées par l'imagination et à les déconstruire en dévoilant leurs artifices. Les tableaux libertins cherchent d'abord à mettre en évidence les détails physiques, la scène étant décrite de façon plus rapprochée que dans les tableaux fantastiques. Les tableaux fantastiques sont quant à eux décrits avec une certaine distance et dans la pénombre. Ces étranges épisodes ont toujours lieu à minuit chez Potocki. Ils sont entourés d'un plus grand mystère, les jeux d'ombres et de lumière cachant certains détails scéniques qui ne sont révélés que plus tard dans le roman.

Dans Pauliska, ou La perversité moderne comme dans le Manuscrit de Saragosse, les tableaux libertins et fantastiques se servent du corps et des sonorités afin de rendre un crescendo d'énergie. Pierre Frantz a souligné que, dans les pièces de Diderot, de Voltaire et de Pixerécourt, on peut observer une série de petits tableaux qui, suivant le principe d'une gradation, contribuent à la mise en place d'une scène finale intense remplie d'émotion pour les personnages et les spectateurs ${ }^{29}$. Cette disposition picturale est ainsi un concept plus esthétique que générique ${ }^{30}$ dont l'effet recherché est d'abord de provoquer une émotion vive chez le public. Loin de se limiter à la représentation d'un seul type de sentiment, le tableau est un concept hybride qui rapproche le théâtre et le roman.

29. Pierre Frantz, op. cit., p. 153-195.

30. Catherine Ramond, op. cit., p. 143. 